\title{
LENS IMPLANTS (PSEUDOPHAKOI) CLASSIFIED ACCORDING TO METHOD OF FIXATION* ${ }^{*}$
}

BY

\author{
C. D. BINKHORST
}

Terneuzen, Netherlands

LENS implants may be classified according to the position of the optical part (posterior chamber lens, anterior chamber lens, pupillary lens), the material used (acrylic, silicate glass, quartz, one-piece lens, two-material lens), and the method of fixation (Binkhorst, 1962a, b). As fixation is obtained by contact of the haptic part of the pseudophakos with the ocular tissues, the subsequent stability of the implant and the integrity of the ocular tissues depend on its design and function. Accounts of the work of surgeons mentioned below, with the exception of Brown and Fedorov, are given by Choyce (1964).

I. Anterior Chamber Fixation (Strampelli, Choyce, Dannheim, Boberg-Ans, Brown, etc.)

The haptic part is held in place in the anterior chamber angle, and is either non-perforating and rigid (Strampelli, Choyce, Boberg-Ans), non-perforating and elastic (Dannheim) (Fig. 1A), perforating (Strampelli, Choyce) (Fig. 1B), or intrascleral (Strampelli, Brown) (Fig. 1C). The optical part is always located in the anterior chamber.

A



B

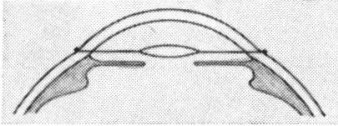

C

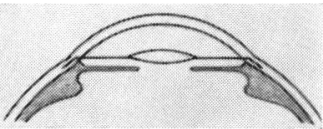

FIG. 1.-Anterior chamber angle fixation.

(A) Non-perforating rigid (Strampelli, Choyce, Boberg-Ans, etc.) or non-perforating elastic (Dannheim).

(B) Perforating (Strampelli, Choyce).

(C) Intra-scleral (Strampelli, Brown).

II. Iris Fixation (Binkhorst, Epstein, Fedorov) (Fig. 2 opposite).

The haptic part is held in place mainly by the iris diaphragm. Even after intracapsular cataract extraction the pseudophakos may also be supported to a small extent by the vitreous body as well. The design varies widely from bulky peripheral formations to fine wire loops. Fixation is either of the iris-clip type (Binkhorst, Epstein) (Fig. 2A, B) or of the iris-cross type (Epstein, Fedorov) (Fig. 2C, D, E). For stability iris-clip fixation has slight advantages over cross fixation. The optical parts may lie entirely in the anterior chamber (Binkhorst $\ddagger$, Fedorov), in the pupillary area (Epstein), or in the posterior chamber (Fedorov). When the optical part is entirely in the anterior chamber contact with the iris is minimal and free passage of the aqueous between the implant and the iris is assured.

III. Irido-capsular Fixation (Ridley, Binkhorst, Fedorov) (Fig. 3 opposite).

The iris diaphragm and posterior capsule co-operate to stabilize the implant after an extracapsular cataract extraction.

In Ridley's original design the periphery of the pseudophakos acted as the haptic part (Fig. 3A). The posterior capsule had to be as clear as possible, but for this reason became rather weak. It was necessary to insert the pseudophakos immediately after cataract

* Received for publication October 3, 1966.

+ Address for reprints: see p. 767.

‡ The author'siris-clip pseudophakos was formerly described as a "pupillary lens"(Binkhorst, 1962), but this is not a true description. 
A B $C$



$\mathrm{D}$

$E$


FIG. 2.-Iris fixation.

(A) "Iris-clip lens" (Binkhorst). Clip fixation. Optical part in anterior chamber.
(B) "Collar stud lens" (Epstein). Clip fixation. Optical part in pupillary area.
(C) "Maltese Cross lens" (Epstein). Cross fixation. Optical part in pupillary area.
(D) Fedorov's modification of "iris-clip lens". Cross fixation. Optical part in anterior chamber.
(E) Fedorov's modification of "iris-clip lens". Cross fixation. Optical part in posterior chamber.

extraction as otherwise the irido-capsular cleft would shrink so that it would not receive the bulky haptic part. This shape requires rather extensive contact between the iris and the implant. The optical part is located in the posterior chamber.

In a recently-described new design of irido-capsular pseudophakos (Binkhorst, 1967), two wire loops form the haptic part (Fig. 3B), and the optical part is entirely located in the anterior chamber, giving free access to the aqueous through the pupil. The central area of the posterior capsule should be as clear as possible, but it is useful to leave a few cortical remnants behind the iris, which will later give rise to the formation of a Soemmerring's ring-i.e. a reinforcement of the capsular membrane. Irido-capsular adhesions embedding parts of the wire loops help to stabilize the implant and prevent undesirable mydriasis. This pseudophakos can be inserted either immediately after cataract extraction or at a second operation. There is minimal contact between iris and implant and the posterior capsule remains intact.
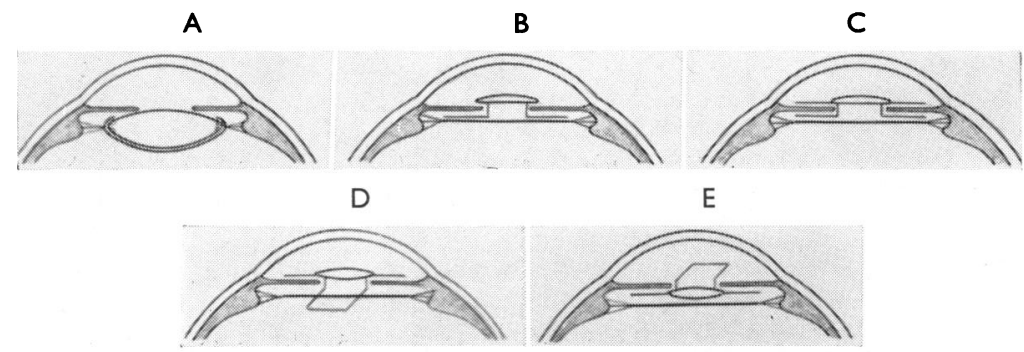

$\mathrm{F}$
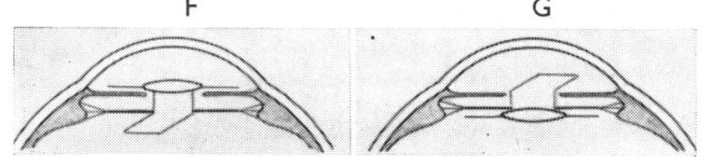

Fig. 3.-Irido-capsular fixation.

(A) Ridley's original "posterior chamber lens". Posterior capsule intact.

(B) "Irido-capsular lens" (Binkhorst). Optical part in anterior chamber. Posterior capsule intact.

(D) "Iris-clip lens" with irido-capsular fixation (Binkhorst). Optical part in anterior chamber. Posterior capsule intact.

Posterior capsule intact.

(E) Fedorov's modification of "iris-clip lens" with irido-capsular fixation. Optical part in posterior chamber. Pos-

terior capsule intact.

(F) Fedorov's modification of "iris-clip lens" with irido-capsular fixation. Optical part in anterior chamber. Posterior capsule perforated.

(G) Fedorov's modification of "iris-clip lens" with irido-capsular fixation. Optical part in posterior chamber. Posterior capsule perforated.

Iris implants such as the author's "iris-clip lens" or Fedorov's modification thereof (Fig. 3C, D, E) can also be used with an intact capsular membrane, the optical part being 
located in the anterior chamber (Binkhorst, Fedorov) or in the posterior chamber (Fedorov).

Another type of irido-capsular fixation has been introduced by Fedorov whereby the pseudophakos is held in place by the irido-capsular diaphragm. The optical part is located either in the anterior chamber or in the posterior chamber (Fig. 3F, G). The pigment epithelium layer of the iris in this technique is protected by the capsular membrane, but the posterior capsule has to be perforated before insertion.

\section{Capsular Fixation (Fedorov)}

Fedorov has also introduced a modification of Binkhorst's "iris-clip lens" which is entirely supported by the capsular membrane, the optical part being located either in front of the membrane or behind it (Fig. 4A, B). He rightly claims that the capsular membrane is the ideal support for a pseudophakos, as it has neither innervation nor blood vessels, and is not covered by endothelium or epithelium. In inserting the pseudophakos, however, the capsular membrane has to be perforated, and Fedorov himself anticipates considerable difficulties during implantation.

A B

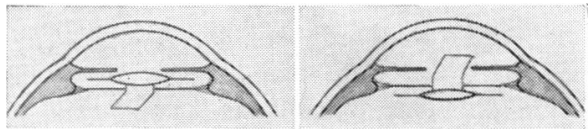

FIG. 4.-Capsular fixation

(A) Fedorov's modification of "iris-clip lens" with capsular fixation. Optical part in front of capsular membrane.

(B) Posterior capsule perforated.

(B) Fedorov's modification of "iris-clip lens" with capsular fixation. Optical part behind capsular membrane. Posterior capsule perforated.

\section{REFERENCES}

BinkHORST, C. D. (1962a). Brit. J. Ophthal., 46, 343.

(1962b). An. Inst. Barraquer, 3, 562.

(1967). Brit. J. Ophthal., 51, 767.

Brown, C. A. Personal communication.

CHоYCE, D. P. (1964). " "Intra-ocular Lenses and Implants". Lewis, London.

FEDOROV, S. Personal communication. 